\title{
Constancy of population parameters for life history and floral traits in Raphanus sativus $L$. I. Norms of reaction and the nature of genotype by environment interactions
}

\author{
SUSAN J. MAZER \& CHARLES T. SCHICK \\ Department of Biological Sciences, University of California, Santa Barbara, California 93106, USA
}

\begin{abstract}
We observed norms of reaction for life history and floral traits in Raphanus sativus L. (wild radish: Brassicaceae) among genotypes raised in three planting densities. In the greenhouse, we used a nested breeding design to produce $\mathrm{F}_{1}$ seed representing 60 maternal plants and 15 pollen donors grown from field-collected seed. Eighteen hundred seeds were grown in three planting densities in an experimental garden. For each individual, we recorded survivorship, germination date, flowering date, petal area, ovule number, pollen production, and the mode of individual pollen grain volume/ flower. Planting density had a strong effect on survivorship, but differential mortality among genotypes was not density-specific. Two-way ANOVAS (block and density as class variables) were conducted on each paternal sibship to detect significant differences among densities with respect to mean phenotype. Among the 15 paternal genotypes, 12 exhibited significantly faster germination in the high-density plots. Three paternal families exhibited significantly delayed flowering at highdensity. Two paternal families exhibited significant effects of density on petal area, but in opposite directions. Two paternal families had significantly lower ovule production at high-density. No paternal families exhibited significant effects of density on pollen production or pollen-grain volume. Strong differences among genotypes with respect to the effects of density on phenotype indicate genetic variation in the plastic response to density for these traits. Three-way ANOVAS of each density treatment measured the effects of block, paternal family and maternal family on phenotype; significant paternal effects indicated the presence of significant additive genetic variance $\left(V_{\mathrm{A}}\right)$ in the measured trait. The ability to detect $V_{\mathrm{A}}$ and maternal effects nested within paternal genotypes in most of these traits was density-specific.
\end{abstract}

Keywords: genotype-environment interaction, heritability, norms of reaction, phenotypic plasticity, Raphanus.

\section{Introduction}

Knowledge of the relationship between genotype and phenotype in different environments is necessary for accurate predictions of the response to natural selection in species which occupy spatially- or temporallyheterogeneous environments. This knowledge is critical for several reasons. First, if the phenotypic expression by a genotype of fitness-related traits depends strongly on growing conditions (that is, given sufficient phenotypic plasticity) then measures of the heritability $\left(h^{2}\right)$ of fitness may also vary across growing conditions. Accurate predictions of the potential for natural selection to result in phenotypic change in traits related to individual fitness require dependable estimates of the heritability of such traits. So, if the relationship between phenotype and genotype is environmentally determined, then $h^{2}$, and consequently the efficacy of natural selection, may also be environmentspecific. In addition, the presence of additive genetic variation $\left(V_{\mathrm{A}}\right)$ in phenotypic plasticity itself implies that plasticity is a trait open to natural selection, a possibility largely unexplored in wild species (but see Westerman \& Lawrence, 1970).

Depending on the way in which the relationship between genotype and phenotype changes across environments, the identity of the genotype favoured by selection and/or the rate of evolutionary change by 
Genotype x environment interactions

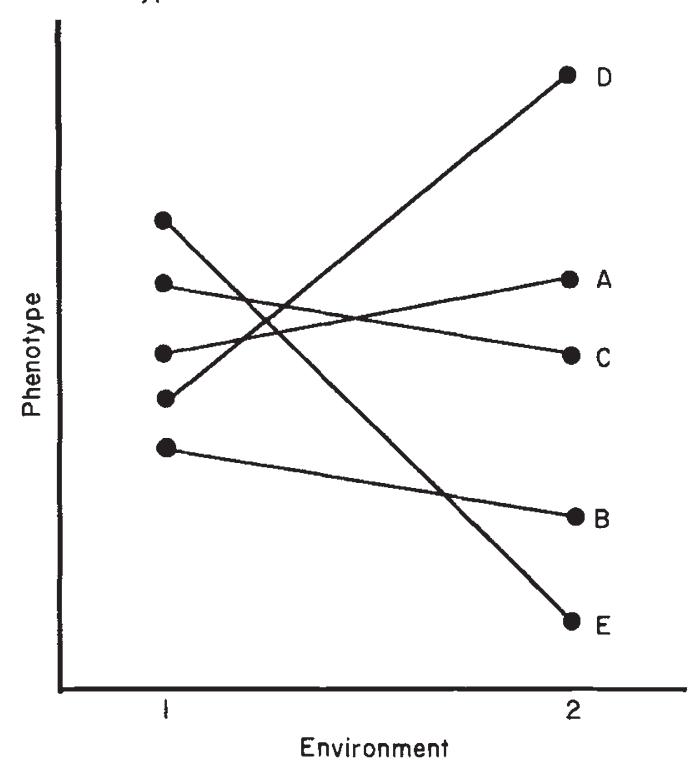

$\begin{array}{lcll} & \begin{array}{c}\text { Magnitude of inter- } \\ \text { genotypic difference } \\ \text { Avs. B }\end{array} & \begin{array}{c}\text { Genotypic } \\ \text { Increases }\end{array} & \begin{array}{c}\text { Direction of } \\ \text { environmental } \\ \text { modification }\end{array} \\ \text { Avs. C } & \text { Remains the same } & \text { Remain the same } & \text { Opposite } \\ \text { Avs. D } & \text { Increases } & \text { Reverse } & \text { Opposite } \\ \text { Avs. E } & \text { Increases } & \text { Reverse } & \text { Same } \\ & & & \text { Opposite }\end{array}$

Fig. 1 Types of genotype $\times$ environment interaction. The relationship between genotype and phenotype may change across environments in two major ways that are not mutually exclusive. First, the magnitude of inter-genotypic variation may change; secondly, the relative phenotypic rank of different genotypes may change across environments. The columns below the illustration indicate four comparisons between pairs of genotypes, and the type of genotype $\times$ environment interaction that they demonstrate.

natural selection may change as well (Fig. 1). For example, given a trait that is consistently, strongly, and positively correlated with individual fitness, if the phenotypic ranks of two genotypes change across environments (compare A versus C, D, or E in Fig. 1), then the genotype favoured by natural selection will also differ between environments. In contrast, if the ranks remain consistent (e.g. compare A versus B, or B versus $\mathrm{D}$ ), but the magnitude of inter-genotypic differences in phenotype increases significantly across environments, then the estimate of heritability and the predicted phenotypic response to selection will increase (given constant phenotypic variance, $V_{\mathrm{P}}$ ) while the genotype favoured by selection remains unchanged. If both the phenotypic ranks and the degree of expressed $V_{\mathrm{A}}$ vary across environments, then both the rate of phenotypic evolution and the particular genotypes favoured in each environment may vary as well.
Secondly, since phenotypic variance due to a $G \times E$ interaction figures prominently in the denominator of the ratio that defines heritability $\left(V_{\mathrm{A}} /\left[V_{G}+V_{E}+V_{G E}\right]\right)$, this source of variance may preclude consistent measures of heritability for traits exhibiting high phenotypic plasticity in spatially or temporally-heterogeneous environments. In such environments, heritability estimates will depend strongly on where, when, and among which genotypes this parameter is measured.

Thirdly, if the relationship between genotype and phenotype varies across environments in a deterministic manner that results in predictable changes in heritability with growing conditions, then it may be possible to identify the conditions under which phenotypic differences among genotypes are most likely to be expressed. If so, then it may be possible to predict the conditions in nature under which evolutionary change by natural or artificial selection may most rapidly occur.

Fourthly, many theoretical models make assumptions about the constancy of parameters such as the heritability of fitness and genetic correlations among fitness components. For example, models of the phenotypic evolution of quantitative characters under multivariate natural-selection assume that genetic and phenotypic covariance matrices remain relatively constant between generations (Lande, 1976, 1979, 1980; see Turelli, 1988 for further discussion). The application of such models to populations in natural habitats requires that the expressed heritabilities of fitnessrelated traits remain constant in spatially and temporally heterogeneous environments. Studies of the nature and strength of $G \times E$ interactions enable evolutionists to evaluate whether these assumptions apply generally to wild species in nature.

Finally, one proposed mechanism for maintaining additive genetic-variance within populations requires the presence of a strong $G \times E$ interaction (Via \& Lande, 1987). If the relative phenotypic rank of genotypes with respect to individual fitness changes across environments, significant $V_{\mathrm{A}}$ may be maintained even in the presence of strong selection and a high heritability of fitness expressed in each environment. Under conditions of frequent shifts in the ranks of different genotypes in distinct microhabitats, different genotypes would be favoured by natural selection in different environments, thus maintaining an array of genotypes in a heterogeneous environment.

This paper is the first in a series that will examine sources of variation in phenotype and genetic parameter estimates for life history, floral characters and reproductive success in Raphanus sativus (wild radish: Brassicaceae). In a second paper (S. J. Mazer \& C. T. 
Schick, in review), we measured the effects of planting density of mean phenotype, heritability estimates and the variance components that comprise heritability. The observed changes in mean phenotype and in heritability with density found in that study may have been due to two non-mutually exclusive processes. First, direct environmental modification of phenotypes (phenotypic plasticity) could have resulted in changes in the mean phenotype of each genotype across densities. This plasticity could also have contributed to variation in estimates of heritability. Secondly, selection via mortality (removing some phenotypes and genotypes from the data set) could have altered $V_{\mathrm{G}}$ and other variance components in a non-random way. In this paper we provide evidence that phenotypic plasticity is more important than viability selection in generating changes in mean phenotype and heritabilty estimates across densities. We present the norms of reaction of individual genotypes and we examine the nature of $G \times E$ interactions in order to describe how phenotypic plasticity altered the relationship between genotype and phenotype in different density treatments. Future papers will focus on: (i) the effects of statistical method, breeding program, and changing environments between generations on heritability estimates; (ii) the degree to which heritability estimates derived from the response to selection are consistent with those derived from the analysis of variance; and (iii) the relationship between phenotypic plasticity and mean fitness across environments.

\section{Study organism}

Raphanus sativus L. (wild radish: Brassicaceae) is an annual, weedy crucifer that occurs in feral populations of disturbed habitats throughout North America, Europe and the Mediterranean; it has been naturalized in California since colonial times (Panetsos \& Baker, 1967). The cultivated garden radish is also identified as $R$. sativus, and feral populations are thought to represent escapes from cultivation. $R$. sativus and its congener $R$. raphanistrum have been the focus of many greenhouse, field and experimental population biology and genetic studies of life history, floral and yield traits (Stanton, 1984a, 1985, 1987a,b; Marshall \& Ellstrand, 1986; Mazer, 1987a,b).

Wild radish is easy to raise under experimental conditions; its short life cycle (3-4 months from seed emergence to the production of ripe seed), large flowers, high flower production and relatively high seed-mass (compared to other rapidly cycling species) allow repeated and easy measurement of reproductive components. Individual plants produce hundreds of seeds in the field and greenhouse, so it is possible to generate enough progeny to replicate genotypes across several treatments. The floral biology and morphology of wild radish lend it to controlled breeding programs. Wild radish exhibits gametophytic-sporophytic selfincompatibility (Putrament, 1960; Lewis et al., 1988); under greenhouse conditions, stigmas do not permit the germination and pollen-tube growth of pollen from the same individual. Moreover, within flowers, dehiscent anthers are physically separated from the receptive stigma, so it is easy to control the application of pollen from desired donors to chosen recipients.

The source population for the genotypes used in this research inhabits an old field on UCSB property adjacent to the Goleta Coastal Wetlands Preserve (GCWP: North of El Colegio Road and east of Los Carneros Road). This field is also the site of the fenced, irrigated experimental garden in which this study was conducted. This close proximity of the parental population to the garden should insure that the phenotypic variation observed among genotypes is typical for the species, and not strongly influenced by observing the genotypes in a novel environment (Service \& Rose, 1985). The seed bank of the garden contained seeds of Avena, Bromus, and other invasive weeds. We did not remove individuals of these species as they germinated during the experiment because we wanted to provide conditions that were as natural as possible. Although evidence of introgression of $R$. raphanistrum germplasm into populations of $R$. sativus is found in many California populations of wild radish (indicated by the appearance of the yellow flower colour characteristic of $R$. raphanistrum: Panetsos \& Baker, 1967), we found no evidence of introgression in this source population.

\section{Materials and methods}

In August 1988, we collected seeds from 100 maternal plants growing in the field adjacent to the GCWP and stored them in the dark at room temperature until December 1988. Seeds were then grown in the greenhouse in 4-inch pots (one seed/pot) until there were 75 synchronously flowering individuals available, each derived from a distinct field-collected plant. Sixty plants were randomly chosen and divided into 15 groups of four individuals which were used as pollen recipients (seed-bearing or maternal plants). Each group was assigned to a different pollen-donor. This is a standard breeding design in which females are nested within males (Comstock \& Robinson, 1948; Hallauer \& Miranda, 1981; Becker, 1984). This greenhouse generation will hereafter be referred to as the parental (or $\mathrm{P}_{1}$ ) generation.

We performed compatibility tests to insure that each 
maternal plant allowed the germination and growth of the pollen of the donor assigned to it (if fruit set did not occur following pollination, the incompatible maternal plant was replaced with a compatible one representing a unique field-collected genotype). Twenty to 30 flowers on each maternal plant were hand-pollinated. Mature fruits were harvested in March 1989. From each of the 60 maternal plants, approximately 30 seeds were randomly chosen to contribute to the $F_{1}$ generation. Nine $8 \times 25$ grids of 200 seeds were designed in which each position was assigned randomly to one of the 60 maternal families. Each grid therefore contained three or four seeds from each maternal family, and three grids were assigned to each of three density treatments. Each maternal family was therefore represented by approximately 10 seeds in each density treatment; each paternal family was represented by about 40 seeds in each density. A total of $1800 \mathrm{~F}_{1}$ seeds were used.

In June 1989, seeds were sown in the irrigated experimental garden. The garden consisted of three blocks, each of which included a high-density, mediumdensity and low-density grid (or plot). The density plots were randomized within blocks. In the high-density plots, seeds were sown $5 \mathrm{~cm}$ apart; in the mediumdensity plots, seeds were sown $10 \mathrm{~cm}$ apart; in the lowdensity plots, seeds were sown $20 \mathrm{~cm}$ apart. One row of field-collected wild-radish seeds was planted around the perimeter of each plot (at the same planting density as the plot) to minimize edge effects.

\section{Measurement of life history and reproductive characters}

In this study, we measured the following fitness-related traits: days to germination, days to flowering, petal area (petal length $\times$ petal width), ovule number, pollen production, and individual pollen-grain volume/flower. Germination date and flowering date have been observed to be strongly correlated with adult plantsize, lifetime flower production and lifetime seed production in $R$. raphanistrum (Mazer, 1987a). In $R$. sativus, Young \& Stanton (1990) found that flower size is strongly associated with pollinator visitation rates and pollen removal, and pollen production is correlated with pollen removal. We included ovule number/flower and individual pollen-grain volume because they serve as relative measures of the allocation of resources to female and male gametophytes at the level of individual flowers.

We recorded the germination date and flowering date for each member of the $F_{1}$ generation. As each surviving plant flowered, we measured one flower per plant for petal area and ovule number; this flower had fully expanded petals. We sampled another flower for the pollen traits; this flower had not yet dehisced its pollen. The two flowers were adjacent to each other when possible.

Pollen-grain number and volume were measured using an Elzone Particle Data analyzer (Devlin, 1988). Flowers were collected in the field, placed in covered Petri dishes, and brought to the laboratory, where the anthers were removed from each flower before they dehisced. All six anthers from each flower were stored in an open plastic-microcentrifuge tube to dry for approximately 1 week. This procedure promotes anther dehiscence. After drying, anthers were transferred to a glass vial containing a known quantity of $2 \%$ $\mathrm{NaCl}$ solution, sonicated for $1 \mathrm{~min}$ to facilitate the release of pollen from the anthers, and analysed $18 \mathrm{~h}$ later. Using the Elzone particle-analyzer we determined the number of pollen grains and the frequency distribution of pollen-grain size (measured in cubic microns) in $0.5 \mathrm{ml}$ aliquots of solution for each sample. For each flower, we determined the total number of pollen grains/flower from the average pollen-count for three $0.5 \mathrm{ml}$ aliquots, weighted by the known total volume of solution. We measured pollen size as the average of the modes of pollen volume obtained from the frequency distributions for the same three aliquots.

To detect significant effects of density on phenotype within each of the 15 paternal families, we used PROC GLM (SAS Institute Inc., 1987) to conduct separate twoway ANOVAS on the progeny of each paternal genotype. These ANOVAS partitioned within-genotype phenotypic variance into effects due to block, density (fixed effects), and the interaction between them. Petal area and ovule number were natural log-transformed prior to analysis; other characters were not transformed because their frequency distributions were unimodal, close to normal, and not improved by transformation procedures.

In addition, for germination date and flowering date, we detected significant effects of density on phenotype across all individuals by conducting a two-way ANOVA on the entire data set to partition variance into effects due to block, density (fixed effects), and the interaction between them. To determine whether natural selection favoured early or late germination (due to possible effects of germination date on survivorship), we compared the mean germinationdates of seeds that survived to flower and of seeds that did not survive in each density treatment.

\section{Analysis of variance: parental effects, heritability estimates, and variance component estimates}

The analysis of variance of the characters expressed in the $F_{1}$ progeny produced by the nested design 
described above can detect significant additive genetic variance among the parental genotypes and provide estimates of narrow-sense and broad-sense heritabilities (Comstock \& Robinson, 1948; Hallauer \& Miranda, 1981; Becker, 1984). The model for a single environment is:

$Y_{i j k}=\mu+m_{i}+f_{i j}+p_{k}+e_{i j k}$

where $Y_{i j k}$ is the phenotype of the $k$ th offspring of the $i$ th pollen donor and the $j$ th female, $\mu$ is the population mean, $m_{i}$ is the effect of the $i$ th father (pollen donor), $f_{i j}$ is the effect of the $j$ th maternal plant mated to (and nested within) the $i$ th male, $p_{k}$ is the plot (or replication) effect and $e_{i j k}$ is the experimental error. Significant paternal effects on progeny phenotype indicate significant additive genetic-variance among the paternal genotypes in the parental generation. This interpretation assumes that the differences among paternal means are due to the effects of paternally inherited nuclear genes, and not due to paternal environmental effects on progeny phenotype generated in the greenhouse (Young \& Stanton, 1990). In this experiment, as paternal genotypes were grown under uniform conditions, and as most of the traits observed in this study are expressed relatively late in ontogeny, the potential for the paternal environment to have a strong effect on progeny phenotype is relatively small. Significant maternal effects include additive genetic and non-additive genetic effects in addition to effects of the maternal environment on progeny phenotype.
We used SAS to detect significant parental effects on progeny phenotype and to estimate variance components. For each density treatment, we used PROC GLM to conduct three-way ANOVAS to partition variance into components due to block (fixed effect), paternal family (random effect) and maternal family nested within paternal effects. Type III sums of squares were used to detect significant effects (at the 0.05 level) of each class-variable independently of the others. We used PROC NESTED to estimate variance components for each trait expressed in each density. Maternal, paternal, error and total-phenotypic variance components were estimated and used to provide estimates of narrowsense and broad-sense heritabilities. Narrow-sense heritability was estimated as four times the paternalvariance component divided by the total phenotypicvariance component $\left(V_{\mathrm{P}}\right)$; broad-sense heritability was estimated as four times the maternal-variance component divided by $V_{\mathrm{p}}$.

\section{Results}

\section{Density effects on survivorship}

Planting density had a very strong effect on juvenile survivorship. Germination rates were high under all treatments ( $>89$ per cent of all seeds germinated); however, juvenile mortality rose sharply with increasing density. In the high-density $(H)$ plots, only 125 of the original 600 seeds survived to flower. In the

Table 1 The means of the days-to-germination (germination date) for all seeds that germinated, for seeds that survived to adulthood, and for seeds that germinated but did not survive to adulthood. Within each sample, shared superscripts indicate that density treatments do not differ significantly with respect to mean germination date at the 0.05 level of significance, as determined by Tukey's test following a two-way ANOVA to detect the effects of block and density (and the interaction between them) on germination date across all genotypes. For each density treatment, the mean germination date of seeds that survived to flower was significantly earlier than the mean germination date of seeds that did not survive (see text)

\begin{tabular}{lllll}
\hline $\begin{array}{l}\text { Population } \\
\text { sample }\end{array}$ & $\begin{array}{l}\text { Density } \\
\text { treatment }\end{array}$ & $\begin{array}{l}\text { Mean } \\
\text { germination } \\
\text { date }\end{array}$ & $\begin{array}{l}\text { Standard } \\
\text { deviation } \\
(\text { s.d. })\end{array}$ & $\begin{array}{l}\text { Sample } \\
\text { size }\end{array}$ \\
\hline All germinated seeds & High & $1.76^{\mathrm{a}}$ & 1.44 & 554 \\
& Medium & $2.81^{\mathrm{b}}$ & 2.70 & 537 \\
& Low & $2.56^{\mathrm{c}}$ & 1.46 & 564 \\
Seeds that survived & High & $1.41^{\mathrm{a}}$ & 0.76 & 123 \\
\multicolumn{1}{c}{ to flower } & Medium & $1.57^{\mathrm{a}}$ & 1.18 & 189 \\
Seeds that did & Low & $2.37^{\mathrm{b}}$ & 1.08 & 326 \\
\multicolumn{1}{c}{ not survive to flower } & High & $1.86^{\mathrm{a}}$ & 1.55 & 431 \\
& Medium & $3.49^{\mathrm{b}}$ & 3.04 & 348 \\
& Low & $2.81^{\mathrm{c}}$ & 1.80 & 238 \\
\hline
\end{tabular}


medium-density $(M)$ plots, 190 survived to flower, and in the low-density plots $(L), 327$ individuals flowered. Natural selection did not favour different genotypes in different plots. Among the 15 paternal genotypes and 60 maternal genotypes, mortality was independent of planting density (Likelihood ratio for paternal genotypes $\chi^{2}=27.93 ; P>0.4681$; model d.f. $=28$; likelihood ratio for maternal genotypes $\chi^{2}=136.67$; $P>0.1153$; model d.f. $=118$ ).

\section{Effects of density on phenotype across all individuals}

We expected that increasing density would be associated with declines in mean phenotype across all individuals, reflecting an inferred decline in resource availability. This prediction was confirmed for development time and for floral traits. Across all individuals, there were strong effects of planting density on germination date and flowering date (the date on which individuals first germinated or began to flower is date 1 ; each subsequent integer represents an interval of 1 day; all densities produced at least one individual that represented the first germination date and flowering date).

Amongst all seeds that germinated (total $n=1655$ ), germination occurred most rapidly in the $H$ treatment (all densities differed significantly from one another; $F$ value for Type III sum of squares $=56.46 ; P<0.0001$ for the density effect on germination date) (Table 1 ). Planting density also had a marginally significant effect on flowering date across genotypes; flowering was delayed at high density $\left(H\right.$ flowering date: $\bar{X}=20.70{ }^{\text {a }}$ s.d. $=12.95 ; n=125 ; \quad$ M: $\quad \bar{X}=16.43,{ }^{b} \quad$ s.d. $=11.47$, $n=190 ; \mathrm{L}: \bar{X}=16.47$, b s.d. $=10.49, n=564$; Tukey's Test; $F$ value for Type III sum of squares $=2.58$; $P<0.07$ for the density effect). Among densities, plots in which plants germinated early ( $H$ plots) flowered late.

The delay in flowering in the high density treatment could have been caused by selection against earlygerminating seeds in these plots in combination with a positive-phenotypic correlation between germination date and flowering date. In fact, within all densities, the correlation between germination time and flowering time was significant and positive; individuals that germinated late tended to flower late (Low-density: $r=0.19 ; \quad$ d.f. $=325 ; \quad P<0.001 ; \quad$ medium-density: $r=0.29$; d.f. $=188 ; P<0.0001$; high-density: $r=0.21$; d.f. $=122 ; P<0.05$ ). If however, the delay at high density was caused by selection against early-emerging seedlings, one would expect the surviving adults in the high-density treatment to be derived from seeds that germinated relatively late. In this study, however, surviving seeds germinated significantly earlier than non-survivors, and the survivors in the high-density treatment germinated particularly early relative to the survivors in the other treatments (Table 1). Within each planting density, the mean germination-date of seeds that survived to adulthood was significantly earlier than the mean germination-date of non-survivors (Kruskal-Wallis test: high density: $\chi^{2}=7.83, P<0.005$; medium density: $\chi^{2}=64.67, P<0.0001$; low density: $\chi^{2}=8.74, P<0.003$ ). Natural selection within all plots favoured early-germinating seeds. The high-density plots simply exhibited a particularly long development time between seedling emergence and flowering.

There was also a statistically significant effect of density on ovule number flower; $H$ plots contained plants with relatively low levels of female gametophyte production/flower (S. J. Mazer \& C. T. Schick, in review). Petal area and pollen production also declined with increasing density, but these declines were not statistically significant. These trends are consistent with the interpretation that $H$ plots provide fewer of the resources necessary for the production of ovules and petals than $L$ plots. There was almost no change in pollen-grain volume with density, suggesting that this trait does not strongly respond phenotypically to the changes in resource levels represented in these plots.

\section{Effects of density on phenotype within paternal genotypes}

In spite of the strong effects of density on phenotype for germination date, flowering date and ovule number/flower across pooled individuals, the quantitative and qualitative effects of density on phenotype are not consistent in strength or direction for all genotypes, but are strongly genotype-specific (Table 2 and Fig. 2). Twelve paternal genotypes exhibited significant differences among density treatments with respect to mean germination-date. None of these 12 , however, exhibited a significant acceleration of germination date between $L$ and $M$; nine genotypes exhibited a significant acceleration of germination date between $M$ and $H$. One genotype exhibited a significant delay in germination between $L$ and $M$; none showed a significant delay between $M$ and $H$.

Effects of density on flowering date were much weaker than the effects on germination date within paternal genotypes. Only three paternal families exhibited significant differences among densities for flowering-date means. None of these families exhibited significant differences between $L$ and $M$ densities; two exhibited a significant delay in flowering between $M$ and $H$ treatments. All three genotypes showed a significant delay in flowering between $L$ and $H$.

Petal area differed significantly among densities within two paternal families, but the effects of density 
Table 2 Means $\pm s$ s.d. of each trait in each density for each paternal sibship. Sample sizes are shown in parentheses. For each trait within each paternal family, shared superscripts $(a, b)$ indicate that means are not significantly different, as determined by Tukey's test following a two-way ANOVA to detect significant effects of density and block on phenotype. For each two-way ANOVA, the total degrees of freedom for the model, the $F$-value associated with the type III sum of squares for density, and the $P$-value associated with the density effect are reported. $P$-values are reported if Tukey's test indicates significant differences among densities, even if the $P$-value is greater than 0.05 . Petal area and ovule number/flower were natural-log transformed prior to analysis

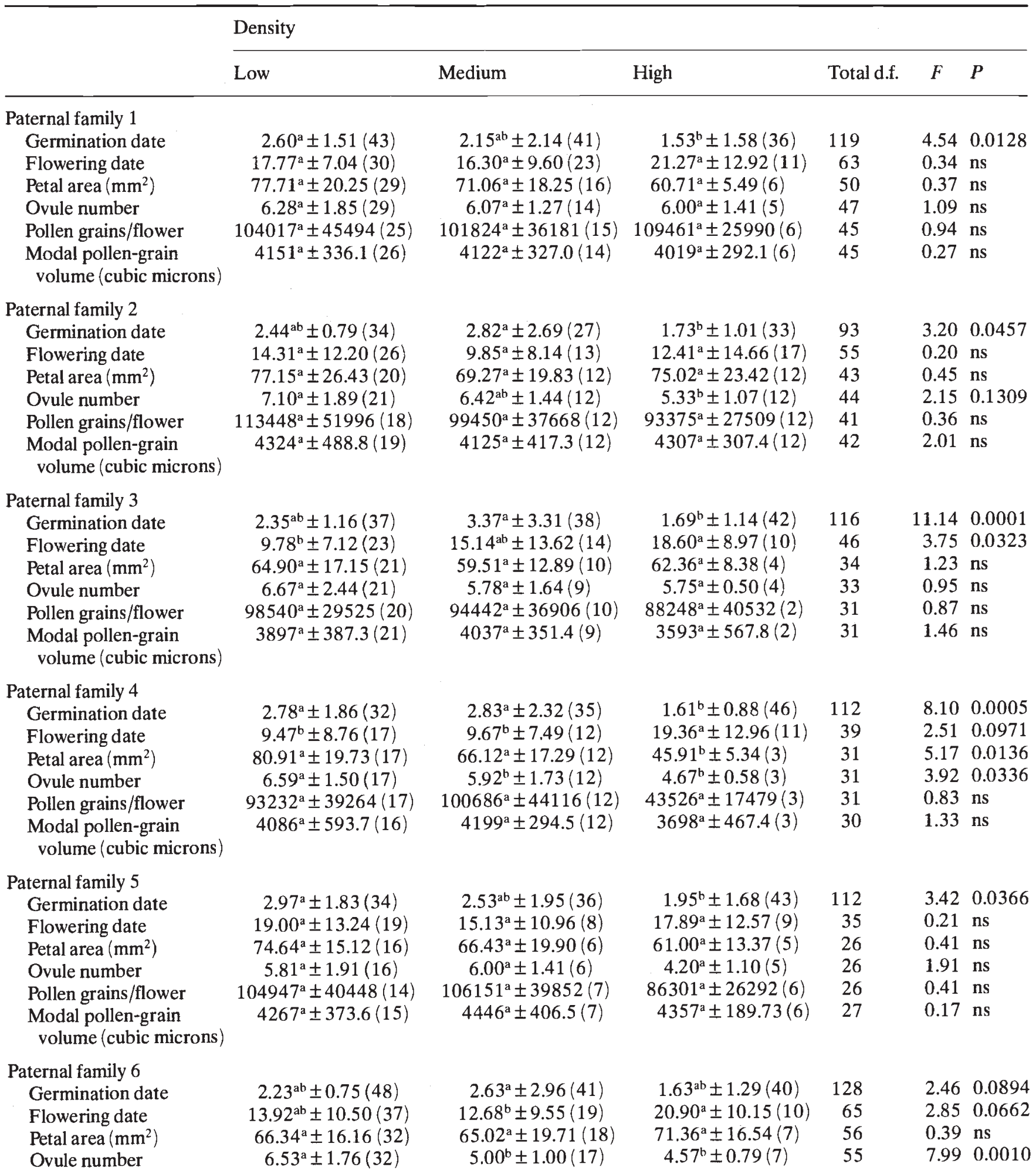


Table 2 Continued.

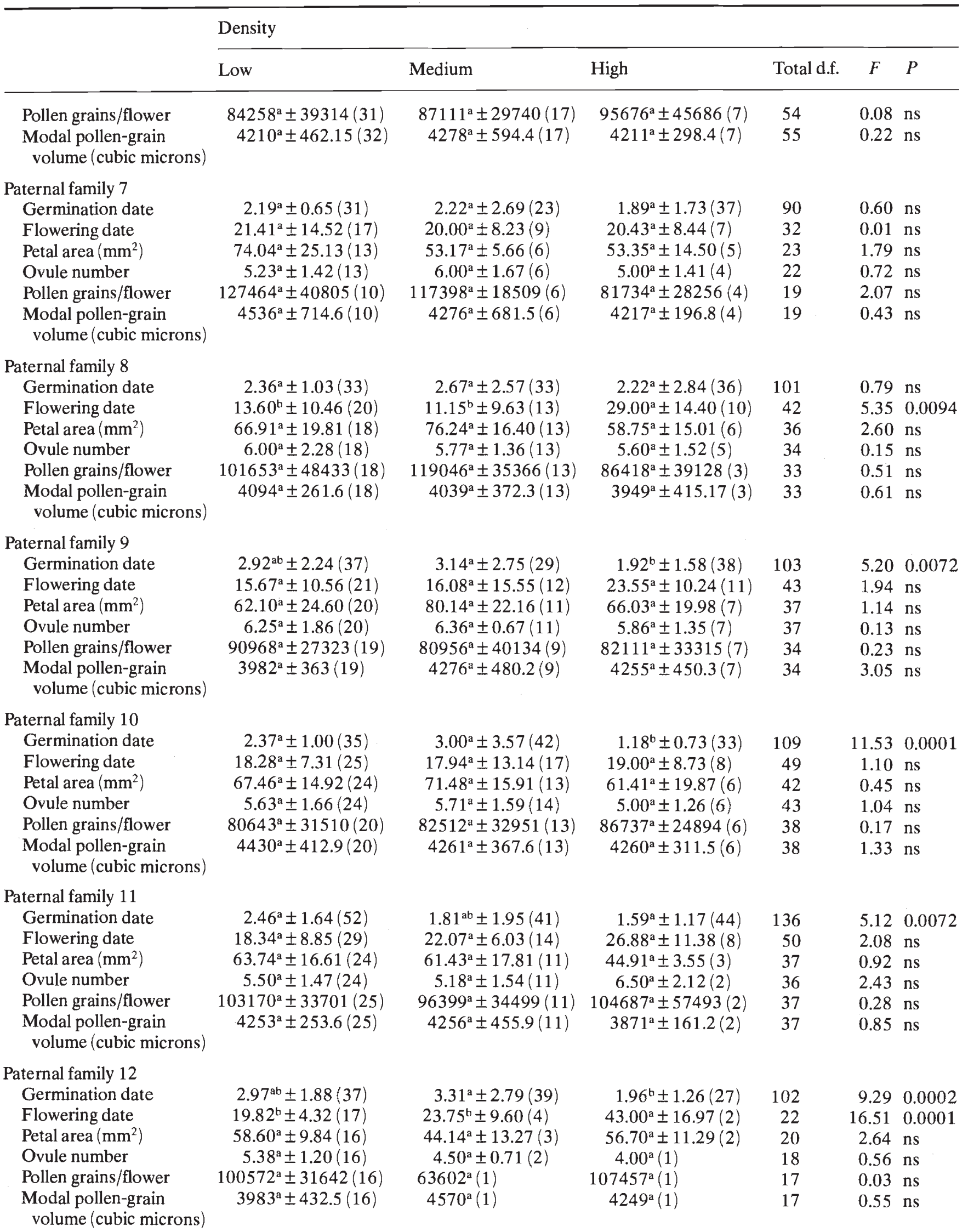


Table 2 Continued.

\begin{tabular}{|c|c|c|c|c|c|c|}
\hline & \multicolumn{6}{|l|}{ Density } \\
\hline & Low & Medium & High & Total d.f. & $F$ & $P$ \\
\hline \multicolumn{7}{|l|}{ Paternal family 13} \\
\hline Germination date & $2.11^{\mathrm{b}} \pm 0.51(38)$ & $3.27^{\mathrm{a}} \pm 2.89(37)$ & $1.73^{\mathrm{b}} \pm 1.04(33)$ & 107 & 5.56 & 0.0051 \\
\hline Flowering date & $24.13^{a} \pm 10.34(15)$ & $30.67^{a} \pm 16.07(9)$ & $49.00^{\mathrm{a}}(1)$ & 24 & 1.29 & ns \\
\hline Petal area $\left(\mathrm{mm}^{2}\right)$ & $66.21^{b} \pm 11.28(14)$ & $88.97^{\mathrm{a}} \pm 18.99(5)$ & (0) & 18 & 12.93 & 0.0029 \\
\hline Ovule number & $5.23^{\mathrm{a}} \pm 1.09(13)$ & $4.40^{\mathrm{a}} \pm 0.55(5)$ & $(0)$ & 17 & 0.35 & ns \\
\hline Pollen grains/flower & $110004^{\mathrm{a}} \pm 40358$ & $119728^{a} \pm 24266(6)$ & $(0)$ & 18 & 0.01 & ns \\
\hline $\begin{array}{l}\text { Modal pollen-grain } \\
\text { volume (cubic microns) }\end{array}$ & $4386^{a} \pm 538.2(13)$ & $4210^{\mathrm{a}} \pm 395.8(6)$ & $(0)$ & 18 & 0.68 & ns \\
\hline \multicolumn{7}{|l|}{ Paternal family 14} \\
\hline Germination date & $2.54^{\mathrm{ab}} \pm 1.04(37)$ & $3.29^{\mathrm{a}} \pm 2.48(34)$ & $2.15^{\mathrm{b}} \pm 1.18(33)$ & 103 & 5.32 & 0.0065 \\
\hline Flowering date & $18.30^{\mathrm{a}} \pm 12.78(20)$ & $14.11^{\mathrm{a}} \pm 9.14(9)$ & $18.83^{\mathrm{a}} \pm 15.12(6)$ & 34 & 0.54 & $\mathrm{~ns}$ \\
\hline Petal area $\left(\mathrm{mm}^{2}\right)$ & $71.31^{\mathrm{a}} \pm 20.48(20)$ & $70.55^{a} \pm 21.78(9)$ & $67.63^{\mathrm{a}} \pm 12.97(4)$ & 32 & 1.08 & ns \\
\hline Ovule number & $5.05^{\mathrm{a}} \pm 1.32(20)$ & $5.22^{\mathrm{a}} \pm 0.97(9)$ & $4.50^{\mathrm{a}} \pm 1.00(4)$ & 32 & 2.75 & ns \\
\hline Pollen grains/flower & $109744^{\mathrm{a}} \pm 25085(20)$ & $103895^{a} \pm 40448$ & $94685^{\mathrm{a}} \pm 28551(4)$ & 32 & 0.26 & ns \\
\hline $\begin{array}{l}\text { Modal pollen grain } \\
\text { volume (cubic microns) }\end{array}$ & $4267^{\mathrm{a}} \pm 373.2(20)$ & $4175^{a} \pm 409.6(9)$ & $4184^{\mathrm{a}} \pm 394.3(4)$ & 32 & 0.46 & ns \\
\hline \multicolumn{7}{|l|}{ Paternal family 15} \\
\hline Germination date & $3.11^{\mathrm{a}} \pm 2.01(36)$ & $3.12^{\mathrm{a}} \pm 2.97(41)$ & $1.70^{\mathrm{b}} \pm 0.88(33)$ & 109 & 5.61 & 0.0049 \\
\hline Flowering date & $18.00^{\mathrm{a}} \pm 9.25(11)$ & $21.36^{\mathrm{a}} \pm 9.51(14)$ & $16.75^{a} \pm 13.89(4)$ & 28 & 1.78 & ns \\
\hline Petal area $\left(\mathrm{mm}^{2}\right)$ & $73.72^{\mathrm{a}} \pm 10.55(11)$ & $73.61^{\mathrm{a}} \pm 18.24(9)$ & $58.25^{\mathrm{a}}(1)$ & 20 & 0.37 & ns \\
\hline Ovule number & $6.09^{\mathrm{a}} \pm 1.81(11)$ & $6.67^{a} \pm 1.41(9)$ & $6.00^{\mathrm{a}}(1)$ & 20 & 0.31 & ns \\
\hline Pollen grains/flower & $134888^{\mathrm{a}} \pm 32518(10)$ & $129450^{a} \pm 61017(8)$ & $110735^{\mathrm{a}}(1)$ & 18 & 0.14 & ns \\
\hline $\begin{array}{l}\text { Modal pollen grain } \\
\text { volume (cubic microns) }\end{array}$ & $4072^{\mathrm{a}} \pm 261.2(10)$ & $4149^{\mathrm{a}} \pm 222.6(8)$ & $4363^{a}(1)$ & 18 & 0.91 & ns \\
\hline
\end{tabular}

were in opposite directions. In family 4 , mean petal area declined significantly between $M$ and $H$ plots, with a marked but non-significant decrease between $L$ and $M$. In family 13 however, which had no survivors at high density, there was a significant increase in mean petal-area between $L$ and $M$.

Ovule number also differed significantly among densities within two paternal families, showing parallel changes in both families. In families 4 and 6 , there was a significant decline in mean ovule-number/flower between $L$ and $M$, and a much smaller decline between $M$ and $H$.

Even more striking than these quantitative differences among paternal genotypes with respect to mean phenotype, are the qualitative differences illustrated by the norms of reaction in Fig. 2. For example, 12 paternal genotypes exhibit a qualitative increase in days-to-germination between $L$ and $M$ planting densities; three genotypes $(1,5,11)$, however, show a qualitative decrease. In contrast, when comparing the phenotypic means of each genotype in the $M$ and $H$ treatments, all genotypes show a qualitative acceleration of germination. With respect to flowering date, eight genotypes $(1,2,5,6,7,8,10,14)$ show an acceleration in flowering between $L$ and $M$ plots; seven show a delay. In the transition between $M$ and $H$, one genotype (15) exhibits qualitative (but non-significant) acceleration of flowering date. For each of these traits, and for each of the two transitions between treatments, there are many instances in which genotypes change rank, as indicated by crossing reaction norms.

The floral traits show similar qualitative differences among paternal genotypes. With respect to petal area, four families $(8,9,10,13)$ increased, and 11 families decreased between $L$ and $M$ treatments. Four different families increased $(2,3,6,12)$ and 11 families decreased in petal area between $M$ and $H$. Ovule number/flower increased in six familes $(5,7,9,10,14$, 15) between $L$ and $M$, but in only one family (11) between $M$ and $H$. The number of pollen grains/flower increased between $L$ and $M$ in six families $(4,5,6,8$, $10,13)$; and in six families $(1,6,9,10,11,12)$ between $M$ and $H$. Finally, mean modal pollen-volume increased in eight families $(3,4,5,6,9,11,12,15)$ 

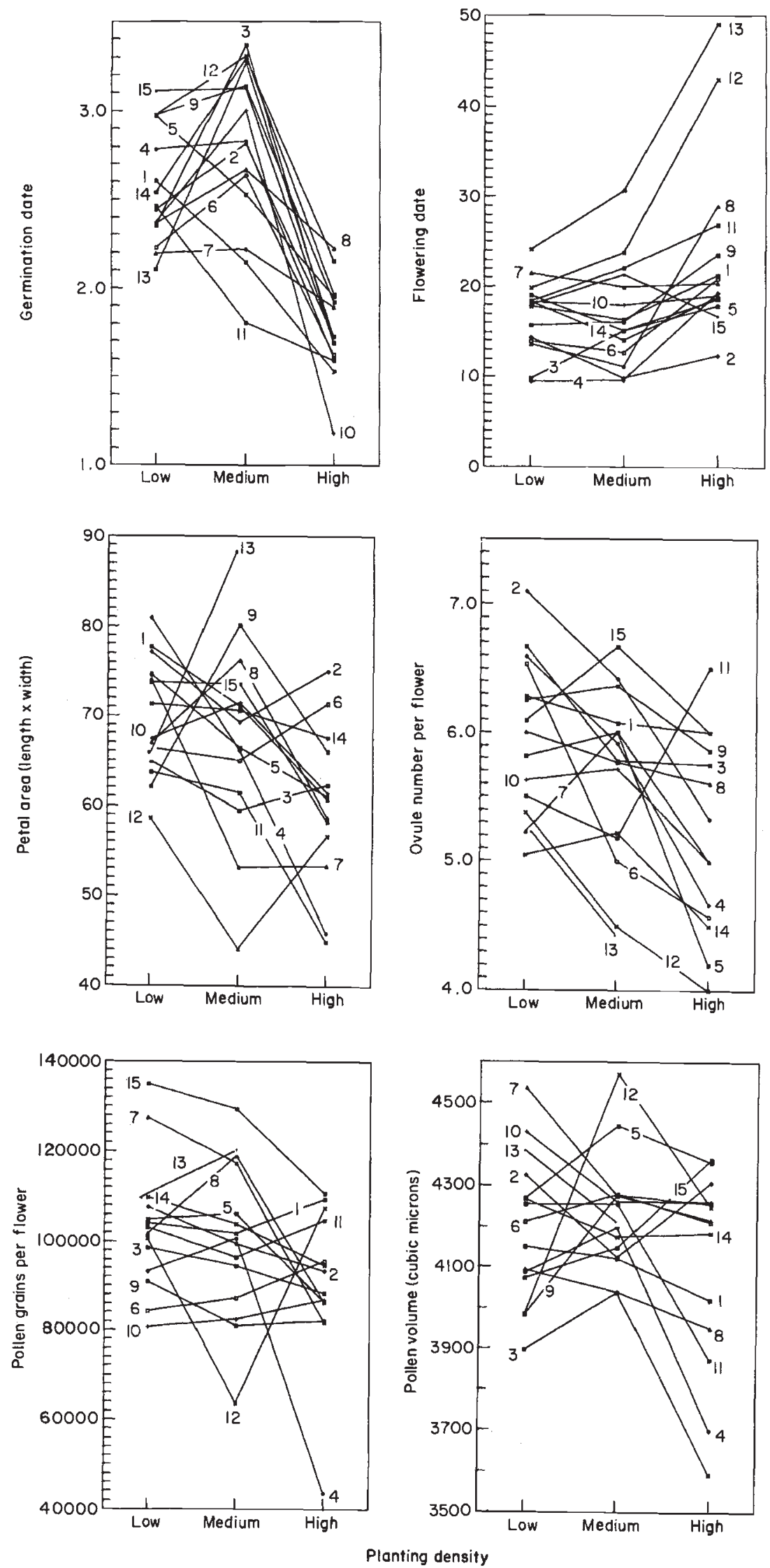

Fig. 2 Norms of reaction for 15 paternal genotypes observed under three planting densities. 
between $L$ and $M$, and in three families $(2,14,15)$ between $M$ and $H$. These differences among genotypes with respect to the direction of phenotypic change with transitions between density treatments, demonstrate that there are qualitative genetic differences in the magnitude and direction of phenotypic plasticity for all traits.

There are many examples of environmental modification of phenotype in which the direction changes as density increases past a threshold. For each trait, there is at least one paternal family that exhibits such a qualitative change in the effect of increasing density on phenotype. For germination date, 12 families germinate most slowly in medium-density plots. For flowering date, only one paternal genotype shows this pattern. For petal area, three families show maximum values at intermediate density, while six families show maximum ovule-number. For pollen production and pollen volume, three and five families respectively, have maximum values at medium density. The tendency for a genotype to show this pattern for one trait does not indicate that it will show this pattern for any other traits.

\section{Density effects on the statistical significance of inter-genotypic variance and heritability estimates}

One way to detect a $G \times E$ interaction is to compare the magnitude of inter-genotypic variation in phenotype expressed in different growing conditions. Comparisons can be made in two ways. The first way is to compare planting densities with respect to the outcome of ANOVAS performed to detect significant paternal or maternal effects on progeny phenotype. Differences among densities in the statistical significance of the paternal effects indicate that the array of phenotypes expressed by the genotypes depends on the environment; that is, there is a significant $G \times E$ interaction. The second way is to compare heritability estimates among planting densities.

Table 3 shows that estimates of narrow-sense $h^{2}$ differ significantly among treatments, but in no clear pattern. Six of the trait/density combinations are associated with narrow-sense $h^{2}$ estimates which indicate that $V_{\mathrm{A}}$ is significantly greater than zero; that is, the three-way nested ANOVA to detect maternal, paternal and block effects detected significant paternal

Table 3 Narrow-sense and broad-sense heritability estimates were derived from paternal and maternal variance components. For narrow-sense heritability estimates, asterisks indicate estimates for which a nested analysis of variance (four maternal plants nested within each male) detected significant paternal effects on progeny phenotype, independently of maternal effects and density effects. For broad-sense heritability estimates, asterisks indicate values for which the ANOVA detected significant maternal effects on progeny phenotype, independently of paternal effects and density effects. Asterisks correspond to the significance levels of the paternal or maternal effects: ${ }^{*} P<0.05 ;{ }^{* *} P<0.01$. Heritability estimates were considered to be zero when variance components were estimated to be negative numbers

\begin{tabular}{|c|c|c|c|c|c|c|}
\hline \multirow[b]{2}{*}{ Density } & \multicolumn{3}{|c|}{ Narrow-sense heritability } & \multicolumn{3}{|c|}{ Broad-sense heritability } \\
\hline & Low & Medium & High & Low & Medium & High \\
\hline \multicolumn{7}{|l|}{ Trait } \\
\hline $\begin{array}{l}\text { Germination } \\
\text { date }\end{array}$ & 0.02 & $0.005^{*}$ & 0.01 & $0.29^{* *}$ & 0.05 & 0.04 \\
\hline $\begin{array}{l}\text { Flowering } \\
\text { date }\end{array}$ & $0.35^{*}$ & $0.49^{* *}$ & 0.10 & 0 & 0.22 & $1.28^{* *}$ \\
\hline Petal area & 0.12 & 0.30 & 0.35 & $0.57^{* *}$ & 0.37 & 0 \\
\hline Ovule number & 0.19 & $0.38^{*}$ & 0.16 & 0.34 & 0 & 0.61 \\
\hline $\begin{array}{l}\text { Pollen grains/ } \\
\text { flower }\end{array}$ & 0.24 & 0.23 & 0.15 & 0.05 & 0 & 0 \\
\hline $\begin{array}{l}\text { Modal pollen- } \\
\text { grain volume }\end{array}$ & $0.40^{*}$ & 0 & $0.68^{*}$ & 0 & 0.38 & 0 \\
\hline
\end{tabular}


effects on progeny phenotype (and the entire model explained a significant proportion of total phenotypicvariance). Three of the six traits show the highest heritability estimates in intermediate densities. Broad-sense heritabilities also differ markedly among density treatments, but in a different pattern (Table 3). Three of these $h^{2}$ estimates indicate trait/density combinations for which the nested ANOVA (to detect maternal, paternal and block effects) detected significant maternal-effects. There are some very large values of broad-sense $h^{2}$ (six values are greater than 0.34), but three of these cannot be interpreted as significantly greater than zero. The discrepancy between broadsense and narrow-sense $h^{2}$ values can in part be explained by the fact that individuals in this breeding design did not serve both as maternal plants and as pollen donors; therefore the narrow- and broad-sense $h^{2}$ estimates are derived from different genotypes.

\section{Discussion}

In this study we measured the effects of planting density on the survivorship, phenotypic expression and norms of reaction of 15 paternal half-sib families with respect to several life-history and floral traits in an experimental garden population of Raphanus sativus. Density had significant effects on phenotypic means for both of the life-history traits measured, but the type of effect on germination date differed from that on flowering date. Germination was accelerated in the high-density treatments relative to medium and low densities, while flowering was delayed at high densities. For floral traits, three of the four characters measured showed declines in value with increasing density, consistent with the interpretation that competitive effects reduce resource levels under high-density conditions. Only one trait, however, (ovule number/flower) declined significantly. The effects of stress (highplanting density) on phenotypic population means appear to be trait-specific and perhaps unpredictable, with the exception that most of the floral traits showed declines in value with increasing stress.

The changes in mean phenotype with changes of density do not appear to be due to non-random mortality of genotypes in the different density treatments. Over all treatments, the differential mortality of both paternal and maternal genotypes was independent of planting density. Natural selection on germination date was not responsible for the subsequent mean differences in flowering date among densities. In all densities, selection favoured individuals that germinated early, and early germination is phenotypically correlated with early flowering. At high densities however, flowering of surviving individuals was significantly delayed relative to low-density plots, even though these survivors had germinated particularly early. With respect to flowering date then, direct environmentalmodification of phenotypes (phenotypic plasticity resulting in a slower development time) was more important than selection in generating the changes in mean phenotype across densities. Environmental modification of phenotype was more important than selection in producing the changes in the mean values of other traits as well. Support for this claim is provided by Fig. 2 which shows frequent and large changes in mean phenotype for paternal half-sib genotypes replicated across planting densities. Because differential mortality of genotypes was independent of density we conclude that the observed changes in mean phenotype and in $h^{2}$ were due primarily to the plastic responses of genotypes across densities.

Changes in the magnitude of heritability estimates do not always correspond to the observed range of phenotypic differences among genotypes (Fig. 2 and Table 3). For example, the narrow-sense heritability of flowering date is not significantly different from zero at high density (Table 3), despite the fact that the range of observed genotype means is far greater in the highdensity treatment than in either of the other two planting densities (Fig. 2). As $h^{2}$ depends critically on $V_{\mathrm{P}}$, which cannot be deduced from inter-genotypic variance alone, there is no necessary correspondence between the magnitude of the differences between genotype means and estimates of heritability.

Interactions between half-sib genotypes and the environment (planting densities) were frequent. For every trait, large changes occurred both in the magnitude of genotypic differences and in the ranks of genotypes with respect to phenotypic means (Fig. 2). In the only other study of $G \times E$ interactions involving density treatments in a natural plant-population (Shaw, 1986), changes in the magnitude of inter-genotype variation and in the relative ranks of maternal genotypes of Salvia lyrata were observed between densities for leaf number.

If these results have general applicability to wild plant-populations, then the response to natural selection should, in many situations depend on local population-density. Density-specific evolutionary responses to selection could occur in two ways. First, differences in the expression of additive geneticvariance among densities could result in differences in the rate of evolutionary change. Secondly, selection could favour different genotypes at different densities if the phenotypic rank of genotypes changes with density while the relationship between phenotype and fitness remains constant. We interpret these results to indicate two possible scenarios for micro-evolution in plant 
populations. First, if consistent differences in density characterize populations on a large geographic scale, then the intensity and response to selection may differ among populations, possibly partly accounting for evolutionary divergence. Secondly, if density commonly differs among stands within populations, then micro-evolution at the population level will be constrained or slowed, and genetic variance should be maintained even under strong selection because of changes in genotypic ranks among stands.

The norms of reaction in Fig. 2 indicate the presence of genetic variation in phenotypic plasticity. For each trait there are paternal half-sib genotypes which exhibit large changes in phenotype with changes in density, indicating high plasticity, and genotypes which remain relatively stable in phenotype across densities, indicating low plasticity. The direction of change in phenotypic expression also commonly differs among genotypes. The conclusion is that phenotypic plasticity in each of these traits is itself a character amenable to change by natural selection in this population of Raphanus sativus. Similar results have been obtained for annual species of Phlox (Schlichting, 1986) and Arabadopsis (Westerman \& Lawrence, 1970; Westerman, 1971a,b,c).

We have demonstrated that mean phenotype and inter-genotypic variation for life history and floral traits are highly sensitive to planting density in $R$. sativus from Santa Barbara, California. If this is a common pattern in wild-plant species then predictions of the response to selection will only be accurate if estimates of $h^{2}$ are determined for commonly occurring environmental states experienced by the species in nature. These changes in the relationship between genotype and phenotype are due primarily to the plastic responses of genotypes across environments, and these responses are not consistent among traits. What is consistent among all traits however, is the presence of genetic variation in phenotypic plasticity. Because we did not measure reproductive output in this study, we cannot deduce whether phenotypic plasticity among these genotypes is an example of developmental flexibility (phenotypic change associated with enhanced mean fitness across environments) or developmental instability (associated with lower meanfitness) (Westerman \& Lawrence, 1970). This is one goal of future studies.

\section{Acknowledgements}

We are grateful for the support of a University of California Faculty Research Grant to SJM. John Damuth, John Endler, Bruce Mahall, Arlee Montalvo, Timothy Prout, Nick Waser and Lorne Wolfe provided many useful discussions and criticisms. Christine Noe and Lisa Meuller provided invaluable assistance in the laboratory and experimental garden.

\section{References}

BECKER, w. A. 1984. Manual of Quantitative Genetics. Academic Enterprises Pullman, Washington. 4th edn.

COMSTOCK, R. E. AND ROBINSON, H. F. 1948. The components of genetic variance in populations of biparental progenies and their use in estimating the average degree of dominance. Biometrics, 4, 254-266.

DEVLIN, B. 1988. The effects of stress on reproductive characters of Lobelia cardinalis. Ecology, 69, 1716-1720.

HALlAUER, A. R. AND MIRANDA, J. B. 1981. Quantitative Genetics in Maize Breeding. Iowa State University Press, Ames, Iowa.

LANDE, R. 1976. Natural selection and random genetic drift in phenotypic evolution. Evolution, 30, 314-334.

LANDE, R. 1979. Quantitative genetic analysis of multivariate evolution applied to brain: body size allometry. Evolution, 33, $402-416$.

LANDE, R. 1980. Genetic variation and phenotypic evolution during allopatric speciation. Am. Nat., 116, 463-479.

LEWIS, D., VERMA, S. C. AND ZUBERI, M. 1. 1988. Gametophyticsporophytic incompatibility in the Cruciferae - Raphanus sativus. Heredity, 61, 355-366.

MARSHALL, D. L. AND ELLSTRAND, N. C. 1986. Sexual selection in Raphanus sativus: experimental data on non-random fertilization, maternal choice, and consequences of multiple paternity. Am. Nat., 127, 446-461.

MAZER, S. J. 1987a. The quantitative genetics of life history and fitness components in Raphanus raphanistrum $\mathrm{L}$. (Brassicaceae): ecological and evolutionary consequences of seed-weight variation. Am. Nat., 130, 891-914.

MAZER, S. J. 1987b. Parental effects on seed development and seed yield in Raphanus raphanistrum: implications for natural and sexual selection. Evolution, 41, 355-371.

PANETSOS, C. A. AND BAKER, H. G. 1967. The origin of variation in 'wild' Raphanus sativus (Cruciferae) in California. Genetica, 38, 243-274.

PUTRAmENT, A. 1960. Studies in self-sterility in Raphanus sativus (L.) var. radicula (DC) Act. Soc. Bot. Pol., XXIV, 289-313.

SAS INSTITUTE, Inc. 1987. SAS/STAT Guide for Personal Computers, Version 6 Edition. SAS Institute Inc., Cary, North Carolina.

SCHLichting, C. D. 1986. The evolution of phenotypic plasticity in plants. Ann. Rev. Ecol. Syst., 17, 667-693.

SERVICE, P. M. AND ROSE, M. R. 1985. Genetic covariation among life-history components: the effect of novel environments. Evolution, 39, 943-945.

SHAW, R. G. 1986. Response to density in a wild population of the perennial herb Salvia lyrata: variation among families. Evolution, 40, 492-505.

STANTON, M. L. 1984a. Developmental and genetic sources of seed weight variation in Raphanus raphanistrum $\mathrm{L}$. (Brassicaceae). Am. J. Bot., 71, 1090-1098. 
STANTON, M. L. 1985. Seed size and emergence time within a stand of wild radish (Raphanus raphanistrum L.): the establishment of a fitness hierarchy. Oecologia, 67, 524-531.

STANTON, M. L. 1987a. The reproductive biology of petal color variants in wild populations of Raphanus sativus L.: I. Pollinator response to color morphs. Am. J. Bot., 74, 178-187.

STANTON, M. L. $1987 \mathrm{~b}$. The reproductive biology of petal color variants in wild populations of Raphanus sativus L.: II. Factors limiting seed production. Am. J. Bot., 74, 188-196.

TURELl, M. 1988. Phenotypic evolution, constant covariances and the maintenance of additive variance. Evolution, 42, 1342-1347.

VIA, S. AND LANDE, R. 1987. Evolution of genetic variability in a spatially heterogeneous environment: effects of genotypeenvironment interaction. Genet. Res., 49, 147-156.
WeSterman, J. M. 1971a. Genotype-environment interaction and developmental regulation in Arabidopsis thaliana. II. Inbred lines; analysis. Heredity, 26, 93-106.

WESTERMAN, J. M. 1971b. Genotype-environment interaction and developmental regulation in Arabidopsis thaliana. III. Inbred lines; analysis of response to photoperiod. Heredity, 26, 373-382.

WESTERMAN, J. M. 1971. Genotype-environment interaction and developmental regulation in Arabidopsis thaliana. IV. Wild material; analysis. Heredity, 26, 383-395.

WESTERMAN, J. M. AND LAWRENCE, M. J. 1970. Genotypeenvironment interaction and developmental regulation in Arabidopsis thaliana. I. Inbred lines; description. Heredity, 25, 609-627.

YOUNG, H. J. AND STANTON, M. L. 1990. Influence of environmental quality on pollen competitive ability in wild radish. Science, 248, 1631-1633. 